\title{
FUENTES DE INFORMACIÓN EN INVESTIGACIÓN SOCIOEDUCATIVA
}

\section{(Information sources on socio-educational research)}

\begin{tabular}{|c|c|c|}
\hline \multicolumn{3}{|c|}{ por } \\
\hline$\underline{\text { Article record }}$ & Alfonso Lorenzo Rodríguez (alfonso.lorenzo@altia.es) & $\underline{\text { Ficha del artículo }}$ \\
\hline About authors & Ana Belén Martínez Piñeiro (amp@uvigo.es) & $\underline{\text { Sobre los autores }}$ \\
\hline HTML format & Esther Martínez Piñeiro (mpineiro@usc.es) & Formato HTML \\
\hline
\end{tabular}

\begin{abstract}
The objective of this work is to be a reference tool for social-educational researchers and staff: a selection of sources of useful information classified in four categories: catalogues, databases, searchers and webs on Internet, and tools for communication between professional colleagues. The electronic addresses necessary for localizing them, are included.
\end{abstract}

\section{Keywords}

Citizenship education; Educational research; Social science research; Information sources; Reference materials; Research tools; Internet; Databases.

\section{INTRODUCCIÓN}

La investigación socioeducativa no puede ser ajena al desarrollo y al impacto de las nuevas tecnologías: investigadores, profesores universitarios y profesionales de la educación social cuentan con nuevas formas de acceso a la información y nuevos canales de transmisión, nuevos soportes informáticos y nuevas soluciones que permiten un análisis de los datos rápido y complejo. Tanto fuentes documentales como herramientas infor-

\section{Resumen}

Este trabajo pretende ser una herramienta de consulta para investigadores y profesionales de la investigación social: una selección de fuentes de información útiles clasificadas en cuatro grupos: catálogos, bases de datos, buscadores y webs en internet, e instrumentos para la comunicación con otros profesionales. Incluye las direcciones electrónicas necesarias para la localización de las fuentes.

\section{Descriptores}

Educación social; Investigación educativa; Investigación en ciencias sociales; Fuentes de información; Materiales de referencia; Herramientas para la investigación; Internet; Bases de datos. 
2000:38). Esta definición permite considerar como fuente de información cualquier objeto o situación que nos transmita algo que, tras su interpretación y elaboración, genera conocimiento.

Concretando más, y en el mismo sentido que aquí queremos dar a la expresión, Fuentes i Pujol (1992:35) define fuente de información como "documento o una institución que (de forma ideal) proporciona respuestas selectivas, fiables, pertinentes, exhaustivas, y oportunas a las preguntas o demandas de un usuario". En el ámbito educativo, Puig (1987:426) define como fuentes "cualquier institución o medio documental que recoja, acumule $\mathrm{y}$, sobre todo, exponga y difunda información pedagógica de cualquier índole". Las fuentes así concebidas son "medios para conseguir la máxima circulación y extensión de la información pedagógica" (Meira, 1997:433).

Las fuentes de información constituyen una plataforma común de la comunidad científica para la difusión de sus conocimientos, y facilitan el flujo y la transmisión de información entre personas, equipos e instituciones interesadas en el estudio de un determinado campo disciplinar, lo que permite al investigador conocer las principales líneas de investigación que se están desarrollando y el progreso del conocimiento sobre el objeto de estudio compartido, acercándose al mismo tiempo a las distintas cuestiones que preocupan a la comunidad científica. Las fuentes documentales son también un reflejo de las demandas sociales a dicha comunidad. Constituyen, en definitiva, un universo teórico, simbólico y experiencial de referencia, en el cual se reconocen e identifican los expertos que comparten un mismo ámbito disciplinar (Meira, 1997).

A la hora de acercarse a esta recopilación de fuentes de información para la investigación socioeducativa el lector debe tener presentes dos aspectos: el ámbito cultural y el momento en el que esta se ha elaborado. El ámbito cultural en cuanto que las necesidades informativas de los investigadores en general, y muy especialmente en cualquier campo social, difieren en función de la cultura en la que su trabajo se desarrolla, y por lo tanto, el ámbito en el que estos seleccionan sus fuentes de información. En nuestro caso, prestaremos atención a publicaciones y recursos europeos (españoles especialmente) y americanos (tanto de Estados Unidos como de América del Sur). Esta pretende, pues, ser una relación selectiva, enfocada a los investigadores de la educación social que desarrollan su trabajo en el estado español; puede también por ello cubrir las necesidades informativas de los profesionales de otros ámbitos geográficos, en especial el latinoamericano, por su estrecha relación con nuestro entorno. En cuanto al aspecto temporal, también el lector tendrá que tener en cuenta el momento de publicación de este trabajo: los recursos a los que nos vamos a referir, en su mayoría electrónicos, podrán desaparecer o modificarse (cambio de denominación, de contenidos, de enlaces...) en breve, y surgirán otros que habrá que añadir a esta selección.

Decimos también que se trata de una relación selectiva, porque, existiendo muchos tipos de fuentes documentales, no nos vamos a referir a todos ellos, ya que, además de que esto implicaría elaborar una lista demasiado extensa y siempre inacabada, consideramos más interesante ofrecer al investigador aquellos instrumentos que le van a servir como intermediarios para localizar y recuperar documentos originales que cubren sus necesidades de información. En concreto, vamos a ceñirnos a:

- Catálogos

- Bases de datos

- Buscadores y webs en Internet

- Instrumentos para la relación con otros/as investigadores/as: listas de distribución y listas de discusión

Tanto catálogos como bases de datos forman parte de los que se denominan docu- 
mentos secundarios, esto es, aquellos que seleccionan y describen documentos, de forma que nos facilitan su localización, al remitirnos a las fuentes originales. Existen varios tipos de documentos secundarios. Hasta hace unos años, y aún en determinados casos en la actualidad, se consultaban habitualmente bibliografías, catálogos, boletines de resúmenes e índices en papel. Hoy, estos documentos impresos están superados por otros de estructura semejante en soporte electrónico: los catálogos y las bases de datos. La diferencia que existe entre ellos es simple: un catálogo describe documentos que forman parte de una colección. Las bases de datos describen documentos que no se hallan en una colección concreta, seleccionados en función de unos criterios previamente determinados, y facilitan el acceso a la información contenida en esos documentos, ya sea mediante descripciones someras (referencias bibliográficas), ampliando estas con resúmenes, o estableciendo índices de todo tipo que facilitan el acceso a esas descripciones. De esta forma, las bases de datos cumplen las mismas funciones que las bibliografías, boletines de resúmenes e índices, con la ventaja de que en ocasiones, además, incluyen todos o parte de esos documentos a texto completo, o el enlace electrónico que nos dirige a ellos.

A pesar de que todavía existen publicaciones en papel que cumplen con eficacia esta función de describir documentos primarios, nos centraremos en los catálogos y las bases de datos electrónicos, al considerar que estos son los instrumentos que hoy tienen más interés para investigadores y estudiantes.

\section{Catálogos}

La norma UNE 50-113-91-/2 (p. 10) define catálogo como "documento secundario que registra y describe documentos reunidos de forma permanente o temporal". Cuando hablamos de catálogos nos referimos, pues, a la descripción de un conjunto de documentos que forman parte de una colección, ya sea una biblioteca, una editorial, una librería...

Las bibliotecas de investigación de cualquier ámbito del conocimiento cuentan hoy en día con catálogos automatizados, en la mayoría de los casos accesible para cualquier persona interesada a través de internet. Las bibliotecas universitarias proporcionan habitualmente este instrumento, básico e imprescindible para investigadores y estudiantes: este es, por lo general, el primer contacto de un estudiante con las fuentes de información, y también los investigadores lo utilizan normalmente como punto de partida para una búsqueda documental.

Para consultar los catálogos de las bibliotecas universitarias españolas no tenemos más que acceder a sus páginas web. Existen, además, dos catálogos colectivos de gran utilidad:

- Rebiun es el catálogo colectivo de la Red de Bibliotecas Universitarias de España. Recoge la información de los catálogos de las bibliotecas universitarias participantes, en dos grupos: monografías (incluye también en algunos casos descripciones de capítulos de libros y artículos de revistas) y publicaciones periódicas. Para cada registro se indica en qué bibliotecas se encuentra el documento, por lo que supone un importante instrumento de localización de los materiales de nuestro interés. Se puede consultar libremente a través de internet (http://rebiun.crue.org/cgi-bin/rebiun).

- El Catàleg Col.lectiu de les Universtats de Catalunya (http://www.cbuc.es/ccuc/) permite consultar conjuntamente los catálogos de las universidades catalanas asociadas, incluyendo monografías y revistas, y con acceso a los sumarios de estas últimas.

En el ámbito educativo destaca, en España, el catálogo del Centro de Información y Documentación Educativas, del Ministerio de Educación, Cultura y Deporte, que describe 
el fondo de la biblioteca, especializado en la legislación, educación comparada, enseñanza primaria, secundaria, profesional, especial, de adultos y universitaria, administración y política educativa. Las ciencias auxiliares de la educación, en especial la sociología y la psicología, están igualmente representadas con una importante colección de obras. Se puede consultar en http://www.mcu.es/bases/spa/bmec/BMEC.h $\underline{\text { tml. }}$

Fuera de España, son relevantes, por el tamaño de sus fondos, lo que las hace de consulta casi inevitable, la Library of Congress, de los Estados Unidos (http://catalog.loc.gov/) y la British Library (http://catalogue.bl.uk/). Para consultar catálogos de bibliotecas europeas, es de gran utilidad Gabriel (http://portico.bl.uk/gabriel), directorio de las bibliotecas nacionales europeas, con enlaces a sus catálogos en internet. Destacamos la Bibliothèque Nationale Française (http://www.bnf.fr/), que da acceso desde su página al catálogo colectivo de Francia; y Myriade, un catálogo colectivo de las publicaciones periódicas de 2.800 bibliotecas y centros de documentación franceses, que se edita en cd-rom.

\section{Bases de datos}

Las bases de datos documentales son, como hemos dicho, fuentes secundarias en formato electrónico. Las mismas bibliografías, los mismos boletines de resúmenes e índices que se consultaban en papel, son, ahora, accesibles mediante un ordenador, con las ventajas que eso supone:

- en las bases de datos los registros se van acumulando a lo largo del tiempo, lo que nos permite, en una sola búsqueda, acceder a la información introducida en ella desde el inicio de su edición; esta ventaja se ha visto incrementada con los nuevos soportes de gran capacidad;

- así, por lo general, la información que contienen los documentos secundarios en papel está incluida con creces en las bases de datos más relevantes;

- a pesar de la cantidad de información contenida, es posible realizar búsquedas muy complejas de una forma asequible, con muchas posibilidades de acotarlas según nuestras necesidades, evitando el "ruido" de la información que no interesa; además, los productores se esfuerzan por proporcionar unas pantallas cada vez más amigables e intuitivas.

La mayoría de las bases de datos que recogemos a continuación no son gratuitas; para su consulta es necesario contactar con sus productores y firmar una suscripción. De ello se encargan habitualmente las bibliotecas universitarias y los centros de documentación especializados.

Para el investigador socioeducativo es interesante conocer no sólo las bases de datos especializadas en su ámbito, sino que en muchas ocasiones tendrá que acudir a otras multidisciplinares pero muy útiles para su búsqueda de información. Esto ocurre con las bibliografías nacionales, bases de datos que recogen todos los documentos publicados en un país. Entre ellas destacamos:

- Bibliografía Nacional Española: Contiene cerca de 440.000 registros bibliográficos de libros de la producción editorial española, ingresados por depósito legal en la Biblioteca Nacional de Madrid desde 1976. Se puede obtener más información en

http://www.bne.es/esp/labipubiblio.htm.

- Bibliographie Nationale Française: Recoge la producción bibliográfica francesa de monografías, publicaciones seriadas y documentos electrónicos, desde 1970, y publicaciones de organismos internacionales (CEE, UNESCO, OCDE...) a partir de 1987. Se consulta directamente a través de los catálogos de la Bibliothèque Nationale de France (http://www.bnf.fr/).

- British National Bibliography: Contiene todas las publicaciones de Gran Bretaña e Irlanda desde 1950. La British Libra- 
ry (http://www.bl.uk/) es el organismo encargado de su publicación, una vez al mes en CD-Rom, y cada semana en papel.

Para conocer documentos que no se pueden encontrar en los circuitos comerciales habituales porque no han sido publicados (lo que se conoce como "literatura gris"), como tesis, tesinas o informes, el investigador puede consultar:

- Digital Dissertations (antes Dissertations Abstracts): Producida por la UMI (University of Microfilms International), proporciona referencias bibliográficas y resúmenes de cerca de millón y medio de tesis y tesinas leídas en Estados Unidos desde 1861, y también en otros países anglosajones desde 1988. UMI complementa este producto con su Dissertation Express, que proporciona las tesis a texto completo previo pago. Se accede a través de la página de UMI (http://wwwlib.umi.com/dissertations).

- Docthèses: contiene referencias de más de 300.000 tesis leídas en Francia desde 1972, con una lista exhaustiva de las bibliotecas que poseen ejemplares de ellas, junto con la información necesaria para solicitar copias. Bibliopolis (http://www.bibliopolis.fr/) produce esta base de datos en cd-rom, con una actualización semestral. También es posible consultar gratuitamente la Bibliographie nationale des thèses en el catálogo Sudoc (Système Universitaire de Documentation, http://www.sudoc.abes.fr/).

- Index to Theses: Accesible previa suscripción, proporciona información sobre las tesis leídas desde 1970 en el Reino Unido e Irlanda (http://www.theses.com/).

- Sigle (System for Information on Grey Literature in Europe): Base de datos que, desde 1980, reúne, analiza y difuende tesis, documentos de trabajo, informes, patentes, preactas y actas de congresos... Elaborada por la European Association for Grey Literatura Exploitation (EAGLE) (http://www.kb.nl/infolev/eagle/frames.ht

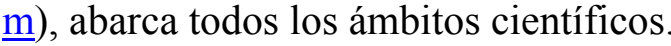

- TESEO: el Ministerio de Educación, Cultura y Deportes español mantiene esta base de datos, accesible gratuitamente a través de Internet (http://www.mcu.es/TESEO/teseo.html), que describe las tesis doctorales leídas y consideradas aptas en las universidades españolas desde 1976. La información procede directamente de la Comisión de Doctorado de las Universidades.

Existen otras bases de datos que recogen información sobre revistas, sin entrar en su contenido, y son muy útiles para saber cuáles pueden resultar más interesantes para un tema de investigación, y en algunos casos para conocer la relevancia de una determinada revista en el mundo científico:

- ISSN: Recoge todas las revistas que cuentan con el número normalizado denominado ISSN (International Standard Serial Number). Permite conocer qué revistas se publican, de forma controlada, en todo el mundo en el ámbito temático en el que trabajamos. Se publica en versión en línea y cd-rom. Para más información se puede consultar http://www.issn.org/.

- Journal Citation Reports: Producida por el ISI (International Scientific Information), recoge y describe las revistas más relevantes, especialmente en el entorno anglosajón. Para ello analiza, de cada revista, datos como el número de veces que fue citada durante un año, la obsolescencia de los artículos que publica, qué revistas cita, etc. Durante el año 2004 todas las bibliotecas de las universidades públicas españolas acceden a esta base de datos a través de la plataforma ISI Web of Knowledge, con la financiación del Ministerio de Ciencia y Tecnología (hoy Ministerio de Educación y Ciencia) y la Fundación para la Ciencia y la Tecnología (FECYT). El acceso a esta plataforma se realiza desde las páginas web de las bibliotecas, o bien

en http://go5.isiknowledge.com/portal.cgi.

- Ulrich's: proporciona información sobre más de 250.000 publicaciones periódi- 
cas, en curso o que ya han dejado de publicarse, de más de 200 países, y de sus editores. Incluye reseñas, direcciones electrónicas y páginas web. Más información y acceso en http://www.ulrichsweb.com/ulrichsweb/.

Ya por último vamos a referirnos a bases de datos que describen artículos y otros documentos, para los que aportan detalles sobre su publicación que permitirán al investigador localizarlos. En algunos casos, como se indican, recogen esos documentos a texto completo. Son multidisciplinares, y por ello también útiles para el ámbito en el que nos centramos, las siguientes:

- Compludoc: Contiene las reseñas de los artículos publicados en una selección de más de mil revistas científicas, en su mayoría españolas, analizadas en la Biblioteca de la Universidad Complutense. La cobertura de los títulos se remonta a 1997, aunque los de ciencias sociales llegan a 1994 e incluyen bastantes revistas extranjeras. Se dividen en cuatro secciones: Área de Ciencias, Área de Ciencias de la Salud, Área de Ciencias Sociales y Área de Humanidades. Su acceso es libre a través de internet (http://europa.sim.ucm.es:8080/compludo c).

- CSIC-ISOC (Consejo Superior de Investigaciones Científicas): El CSIC edita varias bases de datos bibliográficas. ISOC contiene referencias de artículos de alrededor de 2077 revistas, monografías, informes técnicos, actas de congresos, etc., publicadas en España sobre diversas disciplinas de las ciencias sociales y humanidades, desde 1975 hasta la actualidad. Desde 2004 estas bases de datos se pueden consultar, previa suscripción, en http://bddoc.csic.es:8085/ (el acceso gratuito sólo permite consultar tres registros).

- Current Contents: describe el contenido de las revistas con mayor prestigio internacional en ciencias, ciencias sociales y humanidades. Se actualiza semanalmente. Su sección Social \& Behavioral Sciences, se centra en publicaciones de los ámbitos de la comunicación, economía, educación, geografía, derecho, psicología, sociología... Su productor es el Institute for Science Information (ISI), y es otra de las bases de datos accesible para todas las universidades públicas españolas a través de la web http://go5.isiknowledge.com/portal.cgi/.

- Emerald: Recoge 141 revistas electrónicas del ámbito anglosajón, publicadas por la editorial MCB University Press. En algunos casos proporciona el acceso al texto completo. Esta base de datos no es específica del ámbito educativo, sino que las revistas indizadas pertenecen a diferentes campos de las ciencias sociales. Se puede obtener más información en http://www.emeraldinsight.com/.

- Francis: Base de datos realizada por el Institut de l'Information Scientifique et Technique

(http://www.inist.fr/index en.php) de Francia, que tiene como misión coleccionar las investigaciones realizadas en todo el mundo en los ámbitos de las humanidades, ciencias sociales y economía. En el caso de la educación, recoge esta información desde 1975 hasta nuestros días.

- Global Books in Print: Recoge los libros en venta en lengua inglesa. Su ámbito temático es muy amplio, pero su utilidad radica en recoger todo lo que se publica de forma controlada, en inglés, en todo el mundo. Accesible previa suscripción en http://www.globalbooksinprint.com/.

- Periodial Contents Index (PCI): Recoge los sumarios de más de 3500 revistas de ciencias sociales y humanidades publicadas desde 1800, añadiendo en algunos casos sus contenidos a texto completo. $\mathrm{Su}$ interés reside precisamente en que permite el acceso electrónico a artículos antiguos que difícilmente tienen cabida en otras bases de datos. Toda la información sobre esta base de datos se encuentra en http://pci.chadwyck.com/. 
- Social Science Citation Index: base de datos de ISI que contiene referencias de artículos del ámbito de las ciencias sociales, e incluye para cada uno las citas bibliográficas que recoge, otras publicaciones que lo ha citado, y otros artículos con los que comparte alguna referencia bibliográfica. Su estructura permite así conocer la repercusión que un autor o un artículo determinado han tenido en la literatura científica posterior, y acercarnos a autores y obras que de otro modo podrían pasar desapercibidos. Recoge referencias completas de artículos publicados desde 1975, pero en las citas incluye documentos anteriores. Al igual que el resto de las bases de datos del ISI, durante este año se puede consultar desde cualquier universidad pública española en http://go5.isiknowledge.com/portal.cgi o a través de las webs de sus bibliotecas.

- Social Science Source: Editada por EBSCO (http://www.ebsco.com/), incluye artículos a texto completo de unas 140 revistas, e indiza y resume alrededor de 600 . Abarca campos temáticos como trabajo social, psicología, gerontología, justicia criminal, sociología y familia.

- Sociological abstracts: Editada por CSA (Cambridge Scientific Abstracts, http://www.csa.com/), contiene más de 175000 referencias bibliográficas de artículos publicados en unas 2300 revistas desde 1974, y tesis desde 1986. Abarca todos los campos de la Sociología, y disciplinas afines (historia, psicología social, educación social...).

Ofrecemos por último una relación de bases de datos más directamente relacionadas con el ámbito de la investigación educativa:

- Australian Education Index: Producida por ACER Cunninghan Library, del Australian Council for Educational Research (http://www.acer.edu.au/), esta base de datos cita artículos de más de 500 revistas australianas e internacionales, libros, actas de congresos e informes técnicos y de investigación, tesis y legislación, todos ellos relacionados con la investigación educativa, desde 1979 hasta el presente. Excepto las tesis, todos los documentos son accesibles, bien directamente por estar contenidos en la propia base de datos, bien a través del servicio de préstamo interbibliotecario. AEI se edita en línea y en cd-rom.

- British Education Index: Elaborada por la Biblioteca de la Universidad de Leeds, recoge la producción literaria de investigadores del campo de la educación, de alrededor de 300 revistas británicas, informes y actas de conferencias, y textos de la colección Education-line, desde 1976. Se puede obtener (previa suscripción) en papel, Cd-rom, o en línea, en http://www.leeds.ac.uk/bei/bei.htm.

- Canadian Business \& Current Affaires. Education: Base de datos electrónica, equivalente al Canadian Education Index en papel. Es la primera fuente de información sobre educación en Canadá. Recoge referencias de libros y artículos de revistas canadienses, informes de investigación de los gobiernos federal y provinciales de Canadá, tesis, informes de investigación, etc., desde 1976. Se puede obtener más información

en http://www.micromedia.ca/Products Servi ces/CBCAEdu.htm.

- DOCE (Documentos de Educación): Este grupo formado en 1987 por profesores y documentalistas con el objetivo de impulsar la investigación educativa, ha creado varias bases de datos, accesibles desde su página de internet (http://www.eurosur.org/DOCE/) entre las que destacamos su Guía profesional del sector educativo (directorio de empresas, organismos y asociaciones relacionados con la educación), Artículos de prensa (noticias relacionadas con la educación publicadas en la prensa diaria y semanarios especializados), Artículos de revistas (150 revistas de educación), Legislación educativa (disposiciones legales publicadas en el BOE), y Libros profesionales y 
materiales curriculares (libros para la actualización del profesorado).

- E-Psyche: Base de datos editada por Ebsco (http://www.ebsco.com/), que contiene referencias de artículos de revistas, tesis, sitios web, informes técnicos y conferencias de psicología y disciplinas relacionadas con la conducta. Se actualiza semanalmente.

- Educalex: Base de datos elaborada por la editorial Praxis (http://www.praxis.es/) que recoge la principal legislación educativa de ámbito estatal y autonómico, así como una relación de sentencias de diferentes tribunales; añade además comentarios sobre todos los temas relacionados con el sistema educativo, información sobre ayudas y subvenciones, concursos de traslados, calendarios escolares y convenios colectivos.

- Educament: Realizada por la Universidad Rovira i Virgili, contiene referencias de artículos publicados en revistas de educación y psicología. Accesible en la dirección http://teledoc.urv.es/educ@ment.

- Education Index, Education Abstracts, Education Full Text: Bases de datos de H. W.

Wilson

(http://www.hwwilson.com/databases/edu cat.htm) que describe artículos de más de 500 revistas, entrevistas, editoriales seleccionadas, cartas al director, reseñas de libros, películas educativas y software del campo de la educación. La diferencia entre ellas estriba en la profundidad de las descripciones, somera en el Index, con resumen en el Abstracts, y con acceso al texto completo en Full Text.

- Education-line: Elaborada por la Universidad de Leeds, recoge a texto completo literatura gris del ámbito de la educación, desde 1996 hasta nuestros días. Su acceso es gratuito en la dirección de internet http://www.leeds.ac.uk/educol

- Educational Research Abstracts: Recoge referencias de artículos publicados desde 1995 en alrededor de 500 revistas de todo el mundo, y proporciona además ac- ceso al texto completo cuando es posible, a través de la British Library. Su interés se centra en tecnología educativa, educación multicultural, formación y educación técnica, sociología de la educación, gestión educativa y educación especial. Para obtener más información se puede consultar http://www.tandf.co.uk/era/.

- ERIC: Las bases de datos elaboradas por el Educational Resources Information Centre (http://www.eric.ed.gov/), del Education Department de Estados Unidos, son las más conocidas dentro del ámbito educativo, a nivel internacional: CIJE $(\mathrm{Cu}-$ rrent Index to Journals in Education) cubre la literatura sobre educación publicada en más de 775 revistas; y RIE (Resources in Education) incluye proyectos de investigación, conferencias y tesis doctorales. En total, se recogen más de 900.000 citas bibliográficas de documentos desde 1996.

- LEDA: El Ministerio de Educación español elabora esta base de datos que recoge disposiciones normativas en materia educativa, tanto a nivel estatal como autonómico, desde 1970. Se puede consultar gratuitamente en http://leda.mcu.es/

- Psicodoc: Producida por el Colegio Oficial de Psicólogos de Madrid en colaboración con la Facultad de Psicología de la Universidad Complutense de Madrid, incluye resúmenes de artículos de revistas, congresos y libros, publicados en España y América Latina, sobre psicología y materias afines. Se puede consultar libremente con una limitación de acceso a sólo tres registros, o bien a su versión completa para suscriptores, en http://psicodoc.copmadrid.org/.

- Psycinfo: Producida por la American Psychological Association, es la versión electrónica de Psychological Abstracts, que se edita en papel. Contiene referencias y resúmenes de libros, capítulos, tesis y artículos de revistas publicados en más de 50 países desde 1967, siempre en el ámbito de la psicología y ciencias afines. Incluye también investigaciones originales, 
informes de encuestas, estudios de casos... Más información en http://www.apa.org/psycinfo/.

- Psyke: Vaciado de las revistas en español que se reciben en la Biblioteca de la Facultad de Psicología de la Universidad Complutense de Madrid; recoge artículos publicados desde 1985. Se accede libremente a través de internet (http://alfama.sim.ucm.es/isishtm/psyke.ht $\underline{\mathrm{m}})$.

- Redinet (Red Estatal de Bases de Datos sobre Investigaciones Educativas): Recoge referencias de las investigaciones, innovaciones y recursos didácticos producidos en el estado español. Es responsable de su elaboración el Ministerio de Educación, Cultura y Deportes, y se accede a ella libremente

http://www.mec.es/redinet $2 / \mathrm{html}$.

- Reduc es un sistema cooperativo de recopilación, procesamiento y difusión de documentos relevantes en el campo de la educación en la región de América Latina y el Caribe. La base de datos recoge los resúmenes de los documentos, que se encuentran físicamente en microfichas en el Centro Coordinador de Santiago de Chile. Para consultar esta base de datos es necesario asociarse a la Red; toda la información necesaria para ello se encuentra en http://www.reduc.cl/.

- Social Works abstracts: Editada por la estadounidense National Association of Social Workers (http://www.naswdc.org/), contiene referencias de libros, artículos de revista, literatura gris, etc., sobre trabajo social, servicios sociales, pobreza, ancianos, sida, niños y familia, toxicómanos, organización comunitaria, etc.

- UNESBIB: base de datos de la UNES$\mathrm{CO}$ que describe documentos y publicaciones de la UNESCO, de sus oficinas regionales y de los institutos afiliados, así como las colecciones de la biblioteca desde 1972. Incluye documentos de la Conferencia General y del Consejo Ejecutivo, documentos de trabajo, informes de reu- niones y conferencias... Se consulta en http://unesdoc.unesco.org/ulis/index.html. La UNESCO elabora además otros instrumentos de localización de información, como HEDBIB (bibliografía mundial sobre educación superior, http://databases.unesco.org/iau/hedbib/), IBEDOCS (información mundial sobre educación,

http://www.ibe.unesco.org/International/D atabanks/dba.htm), Unesco Thesauro (Tesauro de la UNESCO con 7000 términos, http://databases.unesco.org/thesaurus) y DARE (instituciones, revistas y especialistas en ciencias sociales, http://databases.unesco.org/dare/).

\section{Buscadores y webs en Internet}

No cabe duda que Internet ha venido a revolucionar el mundo de la información, y con él, la investigación en cualquier ámbito científico. La red permite compartir conocimientos, encontrar nuevas ideas, contactar con profesionales de cualquier lugar del mundo, y acceder a una gran cantidad de información. Precisamente este enorme volumen de información hace imprescindible el uso de instrumentos para seleccionar aquella que realmente nos interesa, y a no perdernos en nuestra navegación.

Existen diferentes tipos de herramientas con esta finalidad. Así, Bradley (2003) habla de cuatro categorías: buscadores basados en directorios o índices, buscadores en texto libre, buscadores en lenguaje natural, y meta o multi buscadores. Podemos encontrar distintos tipos de herramientas de búsqueda en http://www.buscopio.net/.

El término directorio se define como "obra de referencia que contiene información dispuesta en orden alfabético, cronológico o sistemático y que permite la identificación o localización de personas, organismos o lugares", tal como se recoge en la norma UNE 50-113-91/2 (p. 7). Esta definición, que nació para los directorios en papel, continúa 
siendo válida para su versión electrónica. Los directorios seleccionan páginas en Internet, los clasifican, y ponen esta clasificación a disposición de los usuarios. Este trabajo de selección y clasificación es humano, no automático, por lo que los directorios habitualmente recogen una cantidad inferior de páginas, pero su indización y recuperación es más precisa. El directorio más conocido a nivel internacional es Yahoo! (http://www.yahoo.es/), pero también existen directorios de gran interés en portales especializados en educación, a los que nos referiremos más adelante.

En cuanto a los buscadores, también recorren las páginas para indizar sus palabras, de forma que podemos localizar aquellas que nos interesan por las palabras que contienen. Este proceso se realiza de forma automática, por lo que alcanzan una mayor cantidad de páginas, pero su precisión es menor. Entre los más utilizados destacamos:

- Google (http://www.google.com/): Ordena los resultados en función del número de enlaces que apuntan a cada documento. Hoy es uno de los buscadores más utilizados. Posee además un directorio, en el que no podemos dejar de destacar Directorio Science $>$ Math $>$ Statistics $>$ Software, que recoge los programas informáticos más utilizados para el análisis estadístico de datos.

- Lycos (http://www.lycos.es/): ofrece una interesante selección de recursos en educación.

- Altavista (http://www.altavista.com/): destaca por su rapidez de respuesta.

\section{- All the web (http://www.alltheweb.com/)}

Existen buscadores a los que podemos interrogar en lenguaje natural, como AskJeeves (http://www.askjeeves.com/); y los llamados metabuscadores o multibuscadores, que utilizan simultáneamente varios buscadores, es decir, no realizan por sí mismos la búsqueda, sino que la lanzan a su vez a varios buscadores, y proporcionan los resulta- dos con un nuevo formato (Müller, 2003). En esta categoría destacamos:

- Kartoo (www.kartoo.com): presenta sus resultados en forma de mapas conceptuales

- Metacrawler

(http://www.metacrawler.com/)

- Compendio (www.compendio.com)

- Ixquick (www.ixquick.com)

Y no podemos olvidar los llamados agentes inteligentes, que además de lanzar la consulta a varios buscadores simultáneamente aportan mejoras como una mayor rapidez de respuesta, filtrado y ordenación de los resultados, criterios de pertinencia propios, posibilidad de salvar y recuperar búsquedas para actualizar sus resultados, etc. Entre ellos destacamos Copernic Agent, cuya versión gratuita se puede descargar desde http://www.copernic.com/.

A pesar de la utilidad de los buscadores, debemos tener presente que no llegan a la totalidad de la información que se encuentra en internet. La llamada internet invisible, compuesta por todas las páginas a las que los buscadores no pueden acceder, constituye habitualmente la fuente de información más interesante para la investigación. Los catálogos de las bibliotecas, las bases de datos, algunos portales institucionales, quedan fuera del alcance de estos buscadores. El investigador puede, para suplir esa carencia, acudir a páginas como www.profusion.com, http://www.internetinvisible.com/, que recopilan recursos de información que no pueden localizarse a través de los buscadores; pero también puede acudir a portales especializados en educación, donde encontrará en la mayoría de los casos información actualizada de su interés y selecciones de recursos, como son:

- Eduso, el portal de la educación social (http://www.eduso.net/): clasifica enlaces relacionados con la educación social. Da acceso además a un chat y un foro de edu- 
cadores, convocatorias, ofertas, bibliografía, revistas de educación social, etc.

- Educaweb

(http://www.educaweb.com/): este portal, de la empresa Educaonline, destaca por su directorio de instituciones educativas y otros recursos de interés, y su agenda de enventos, completa y actualizada.

- Edufind (http://www.edufind.com/): portal que recoge recursos para la educación (programas informáticos, cursos y congresos, centros educativos...).

- The educator's reference desk (http://www.eduref.org/): servicio del Educational Research Information Center, que ofrece una interesante selección de recursos en la red.

Otra forma de acceder a la internet invisible es a través de las páginas de centros de documentación especializados, cuyo instrumentos de búsqueda podremos utilizar. Algunas de las bibliotecas universitarias españolas recogen en sus páginas web selecciones de enlaces de interés en educación muy adecuados a las necesidades de la investigación socioeducativa; destacan las bibliotecas de las universidades de:

- Sevilla (http://bib.us.es/),

- Complutense de Madrid (http://www.ucm.es/BUCM/edu/index.ht $\underline{\mathrm{m}}), \mathrm{y}$

- Barcelona

(http://www.bib.ub.es/www5/5intedu.htm)

Fuera de nuestras fronteras, podemos destacar:

- Cubberley Education Library, de la Stanford University (http://wwwsul.stanford.edu/depts/cubberley/), cuya página contiene una amplia y estudiada selección de recursos de información para la investigación educativa.

- University of Georgia Library (http://triton3.galib.uga.edu/cgibin/homepage.cgi?style $=\&$ id $=$ c192205f$1087155572-1665 \&$ \&c $=1$ ), que posse una completa colección sobre investigación educativa, y mantiene una interesante selección de recursos en Internet.

- Leeds University Library (http://www.leeds.ac.uk/library/), cuyos servicios son de referencia obligada para nuestros investigadores: ya hemos citado sus base de datos Education-line y British Education Index, pero debemos señalar además su catálogo de recursos en internet, su Conference programmes (herramienta de apoyo para organizadores de eventos) y su Education Conference Listing Service, que recoge congresos y otros actos que han tenido o tendrán lugar en el ámbito educativo, no sólo en el Reino Unido, sino también en otros países.

- Miami University Library (http://library.miami.edu): proporciona una completa relación de fuentes de información en diferentes campos, entre ellos educación, psicología y disciplinas afines. Destaca su documento Research methods in social science; an internet resource

list

(http://www.library.miami.edu/netguides/ psymeth.html).

\section{Otros centros de documentación especia- lizados en el ámbito educativo son:}

- Biblioteca Virtual de Educaçao: http://bve.cibec.inep.gov.br/. Se trata de una interesante selección de recursos educativos, con múltiples formas de búsqueda y acceso, elaborada por el INEP (Instituto Nacional de Estudos e Pesquisas Educacionais Anísio Teixeira) de Brasil.

- Centro de Información y Documentación

Científica:

http://www.cindoc.csic.es/. Es el centro de documentación del Centro Superior de Investigaciones Científicas, y proporciona acceso a la base de datos del ISOC, a sumarios de revistas, tesauros, información sobre cursos, congresos, etc.

- Centro de Documentación Especializado en Educación de Adultos para América Latina

(CEDEAL): 
http://atzimba.crefal.edu.mx/cedeal/calida d educacion.htm. Es un centro de información en educación de adultos, que recoge bibliografía y recursos de información clasificados según su temática.

- Centro de Documentación sobre Desarrollo

Comunitario:

http://documentacion.edex.es/default.asp.

Pertenece a Edex, una institución cuyo objetivo es la prevención de las drogodependencias. Su centro de documentación proporciona recursos de información y bibliografía clasificados por temas.

- Centro de Información y Documentación en Educación (CIDE): http://www.mec.es/cide. Es el centro de documentación del Ministerio de Educación, Cultura y Deportes. Da acceso a los catálogos de su biblioteca y archivo, proporciona información y documentos útiles para profesores e investigadores, y una agenda de eventos durante el año en curso.

- Educational Research Information Center (ERIC): http://www.eric.ed.gov/. Sistema de información sobre educación del Departamento de Educación de Estados Unidos. Elabora dos bases de datos de gran importancia para la investigación educativa, y da acceso a otros recursos de interés.

Es importante visitar páginas de instituciones relacionadas con nuestra área de trabajo. En la mayoría de las ocasiones estas permiten conocer sus actividades y participar en algunas de ellas, difunden sus conclusiones y las opiniones de sus expertos, y, al mismo tiempo, dan la oportunidad de contactar con otros investigadores, participar en sus foros de debate, plantear dudas, buscar colaboradores para algún proyecto, etc. Algunas de las instituciones que pueden resultar interesantes para la investigador de la educación social son:

\section{- American College Testing:} http://www.act.org/. Organización estadounidense que proporciona apoyo, información y servicios de gestión de programas educativos.
- American Educational Research Asociation (AERA): http://www.aera.net/. Organización profesional de investigadores educativos de Estados Unidos.

- American Psychological Association (APA): http://www.apa.org. Asociación profesional de psicología de Estados Unidos, cuyas recomendaciones son tenidas en cuenta a nivel internacional. Su página web es una buena fuente de información para temas relacionados con la psicología.

- Asociación Española de Orientación y Psicopedagogía

http://www.uned.es/aeop/.

(AEOP):

- Asociación Interuniversitaria de Investigación

Pedagógica: http://www.uv.es/aidipe. Esta asociación publica dos revistas, organiza congresos, reuniones científicas, mantiene un foro de discusión, proporciona recursos de información...

- Australian Association for Research in Education (AARE): http://swin.edu.au/aare. Su página web da acceso a sus publicaciones, congresos, tesis doctorales australianas relacionadas con la investigación educativa, etc.

- Australian Council for Educational Research (ACER): http://www.acer.edu.au/

- British Educational Research Association (BERA): http://www.bera.ac.uk/. Asociación con sede en Notts (Inglaterra) encargada de la edición de British Educational Research Journal; tiene como objetivo fomentar una cultura de la investigación en el ámbito educativo.

- Center for Educational Research and Innovation (CERI): http://www.oecd.org/. Esta organización forma parte de la OECD (Organisation for Economic Co-operation and Development), y pretende fomentar el diálogo sobre educación, fomentando proyectos de investigación educativa.

- Consejo de Europa: http://www.coe.int/ . Es interesante visitar el apartado de cooperación para la educación de la web del Consejo de Europa, donde se exponen los programas educativos que se están des- 
arrollando, estándares educativos, información sobre conferencias, publicaciones, etc.

- Consejo Mexicano de Investigación Educativa: http://www.comie.org.mx . Entre las acciones que el COMIE realiza están la organización, cada dos años, del Congreso Nacional de Investigación Educativa, y la edición de la Revista Mexicana de Investigación Educativa.

- Consortium of Institutions for Development and Research in Education in Europe

(CIDREE): http://www.cidree.org/about_profile.html.

Esta organización de reconocido prestigio en Europa, trata de promocionar la calidad educativa, proponiendo ideas y actividades, y proporcionando información a sus miembros.

- CTI Statistics software reviews: http://www.stats.gla.ac.uk/cti/activities/rev iews/alphabet.html. Lista de recursos estadísticos que ofrece la universidad de Glasgow.

- Departamento de Didáctica e Investigación Educativa y del Comportamiento, de la Universidad de La Laguna: http://webservices.ull.es/departamentos/di dinv/dibmusic/i-explor/pgmarcos.htm.

Proporciona información sobre las líneas de investigación del departamento, las tesis leídas, y una interesante selección de enlaces sobre tecnología educativa.

- Departamento MIDE de la Universidad Complutense de Madrid: http://www.ucm.es/info/mide/. Además de los datos básicos del departamento, su página proporciona recursos de información para la investigación educativa, y facilita y anima al contacto con los investigadores del departamento.

- Departamento de Didáctica y Organización Escolar y Métodos de Investigación y Diagnóstico en Educación de la Universidad de Sevilla: http://www.us.es/ddoe. Contiene enlaces a sus grupos de investigación, con amplia información sobre investigación educativa.

- European Association of Research on Learning and Instruction (EARLI). http://www.earli.eu.org/. Se trata de una asociación para el intercambio de opiniones e información.

- Easter Educational Research Association: http://www.govst.edu/eera. Asociación estadounidense que tiene como objetivo fomentar la investigación educativa; una de sus divisiones trata los temas de medición, estadística, evaluación e investigación.

- Europa: la Unión Europea en línea: http://europa.eu.int/.

- European Educational Research Association: http://www.eera.ac.uk/. Tiene como objetivos el intercambio de información entre investigadores europeos y la elaboración de propuestas políticas; edita la revista European Educational Research Journal. Forma parte de ella la AIDIPE.

- Eurydice: http://www.eurydice.org/. Es la red europea de información en educación; tiene como objetivos favorecer el intercambio de información entre los reponsables políticos europeos en materia educativa, e informar a todas las personas interesadas. Proporciona una interesante relación de referencias bibliográficas, datos estadísticos, estudios comparativos...

- Florida Educational Research Association: http://www.tfn.net/fera/. Asociación profesional que edita la revista Florida Journal of Educational Research, y tiene la misión de impulsar la investigación educativa en el estado de Florida (Estados Unidos).

- Freestatistics.altervista.org: recopila programas informáticos matemáticos y estadísticos, además de otros recursos relacionados con la estadística. Permite descargar los programas que son gratuitos.

- Grupo de Tecnología Educativa: http://gte.uib.es/. Grupo de investigación de la Universitat de les Illes Balears, que 
se dedica a las nuevas tecnologías de la información aplicadas a la educación.

- Hegoa: Instituto de Estudios sobre Desarrollo y Cooperación Internacional de la Unviersidad del País Vasco: http://www.hegoa.ehu.es/topics/intro/. Interesante página sobre esta institución, que incluye referencias a la educación para el desarrollo y consultas a su centro de documentación.

- Institut National de Recherche Pedagogique: http://www.inrp.fr. Instituto dependiente del ministerio encargado de la educación superior en Francia, creado en 1993 para coordinar y desarrollar la investigación educativa a todos los niveles, la formación del profesorado, la elaboración de recursos educativos... Es el organismo encargado de la biblioteca y el museo nacionales de la educación.

- Institute of Education Science: http://www.ed.gov/about/offices/list/ies/in dex.html. Instituto del Departamento de Educación del gobierno de Estados Unidos, creado en 2002, del que depende el Educational Research Information Center, y el National Center for Educational Research.

- Institute of Education, University of London: http://www.ioe.ac.uk. Instituto fundado en 1902 como centro de investigación educativa y formación del profesorado. Da acceso a sus proyectos de investigación en curso.

- Institut de Recherche et de Documentation Pédagogique: http://www.irdp.ch/index.html. Instituto dependiente de la Université de Neuchâtel de Suiza, y que se encarga de desarrollar y coordinar investigaciones sobre sistemas de formación, didácticas, reformas educativas y aplicación de nuevas tecnologías a la educación.

- Instituto de investigación y Desarrollo Educativo de la Universidad Autónoma de Baja California (México): http://iide.ens.uabc.mx/. Este instituto, responsable de la Revista Electrónica de
Investigación Educativa, mantiene una interesante página web que proporciona amplia información sobre sus proyectos y actividades, así como acceso a su centro de información.

- Instituto Nacional de Evaluación y Calidad del Sistema Educativo: http://www.ince.mec.es/. Organismo que depende del Ministerio de Educación español, que tiene como objetivo evaluar el sistema educativo.

- International Development Research Center: http://web.idrc.ca/ev_en.php. Organismo gubernamental de Canadá que tiene como objetivo ayudar a los países subdesarrollados a buscar soluciones para sus problemas sociales, económicos, y medioambientales, a través de la investigación.

- International Institute for Qualitative Methodology:

http://www.ualberta.ca/ iiqm. De la Universidad de Alberta (Canadá); proporciona una relación de recursos para la investigación cualitativa

- National Center for Education Statistics: http://nces.ed.gov/. Recoge y analiza datos relacionados con la educación en Estados Unidos y otros países.

- National Council for the Social Studies. http://www.ncss.org/. La misión de esta institución es proporcionar servicios y soporte para los educadores de estudios sociales. Fundada en 1921, tiene miembros en 70 países; sirve como una organización para profesores de todos los niveles de historia, geografía economía, política, sociología, psicología, antropología y derecho.

- National Council on Measurement in Education: http://www.ncme.org. Organización profesional con sede en Washington, que tiene entre sus misiones impulsar la evaluación educativa y elaborar instrumentos de medición de la educación.

- National Foundation for Educational Research: http://www.nfer.ac.uk. Organización independiente inglesa que tiene 
como misión analizar y difundir información relativa a la investigación educativa. Mantiene una base de datos de proyectos de investigación denominada Ceruk.

- Oficina Internacional de Educación: http://www.ibe.unesco.org/index.htm.

Oficina de la Unesco que tiene como funciones principales "observar, promover el diálogo sobre políticas de la educación, reforzar el ámbito de desarrollo de competencias, y difundir información".

- Organización de Estados Iberoamericanos para la Educación, la Ciencia y la Cultura: http://www.oei.es/. Se trata de un organismo de países iberoamericanos para fomentar la cooperación en el ámbito educativo. Su página web proporciona información muy valiosa para investigadores y profesores, como un apartado de eventos y un índice de revistas de interés.

- Science Plus Group: http://www.scienceplus.nl. Presenta un catálogo de programas informáticos ordenados alfabéticamente, útiles para la investigación social.

- Scottish Educational Research Association: http://www.sera.ac.uk/. Esta asociación está formada por profesionales de la educación de todos los niveles, y tiene como objetivo promover la investigación educativa, y su aplicación práctica.

- Sociedad Española de Pedagogía: http://www.uv.es/ soespe. Organismo responsable de la publicación de la revista Bordón; organiza cursos, conferencias, congresos, promueve estudios, elabora informes, etc., con el ánimo de poner en contacto a los profesionales de la pedagogía españoles.

- Statlib: sistema para distribuir conjuntos de datos y de software estadístico disponible libremente. Pertenece al departamento de estadística de la Carnegie Mellon University.

- UNESCO: http://www.unesco.org/. La Organización de las Naciones Unidas para la Educación, la Ciencia y la Cultura mantiene una completa página en internet con información sobre sus proyectos, acceso a sus documentos, enlaces de interés, bases de datos documentales...

\section{Instrumentos para la relación con otros/as investigadores/as: listas de dis- tribución y grupos de discusión}

Qué duda cabe que toda fuente de información es al mismo tiempo un mecanismo de difusión de esta. Este flujo de información en doble sentido (de entrada y salida) se hace más patente si cabe en las listas de correo electrónico. Estas suponen una rápida forma de comunicación entre un grupo de personas que comparten unos intereses comunes. Para poder formar parte de una determinada lista basta con solicitarlo, y ser aceptado por el moderador. A partir del momento de la suscripción, se reciben todos los mensajes enviados a la lista por todos sus miembros, y en sentido inverso, cualquier mensaje enviado a la lista es recibido por todos los demás. De esta forma se abre un foro de discusión donde todos pueden aportar y recibir información. Existen listas de distribución libres, en las que todos los mensajes enviados son automáticamente distribuidos a todos los participantes, pero también hay listas moderadas, donde una o más personas se encargan de revisar y dar o no el visto bueno a los mensajes enviados a la lista, para asegurar que esta se limita a los temas previamente determinados (Martínez López, 2001).

Los grupos de discusión o de noticias están basados en esa misma filosofía de compartir información y establecer debates, con la diferencia de que los mensajes no se distribuyen, sino que se almacenan en los servidores de noticias, y cualquiera puede consultarlos y participar sin necesidad de suscribirse (Martínez López, 2001).

Existen numerosas listas de distribución y grupos de discusión de temática educativa, y más específicamente centradas en cuestiones de investigación, promovidas por asociaciones, organismos públicos, o profesionales 
interesados en establecer contacto con otros colegas. El servicio de listas de la Red Iris (http://www.rediris.es/list/) proporciona información sobre todas ellas, clasificadas temáticamente; a nivel internacional, podemos consultar

http://www.lsoft.com/lists/listref.html, http://tile.net/, http://www.e-listas.net/. La American Evaluation Association proporciona en su web una buena relación de listas relacionadas con la evaluación y la investigación

(http://www.eval.org/EvaluationLinks/Listse rvs.htm); y para investigación cualitativa, podemos encontrar una relación de listas en http://www.qualitative-

research.net/qf/psde/q-mailing-e.htm. Destacamos las siguientes listas, tomando como referencia a Aliaga y Suárez (1995) y Sandín (2001):

- Aelaction

(http://offchemmath.roshd.ir/ael01/rel/schl serv/actlist.htm): Lista de discusión de Appalachia Educational Laboratory in Charleston, West Virginia, dedicada a la investigación-acción.

- Aera (http://lists.asu.edu/): listas de la American Educational Research Association, en las que se puede obterner información sobre congresos, reuniones, nuevas publicaciones, nuevos programas informáticos...

- AIDIPE-L

(http://www.rediris.es/list/info/AIDIPEL.html): grupo de investigación de la Asociación Interuniversitaria de Investigación Pedagógica, pionera en España en listas sobre investigación educativa.

- EVALTALK

(http://www.eval.org/ElectronicLists/evalt alk.html): Lista promovida por la American Evaluation Association,

- ONLINERSCH: centrada en el estudio y la reflexión sobre ética y metodología de la investigación social.

- Qualitative research on the human Science (http://listserv.uga.edu/archives/qualrs1.html), de la University of Georgia.

\section{Consideraciones finales}

Hemos recogido en este trabajo una selección amplia de fuentes de información de interés para la investigación socioeducativa y para los profesionales de la educación social. Cada investigador, cada profesional, no puede, no debe usar un número excesivamente alto de fuentes de información, lo más recomendable es que escoja aquellas que más se adecúen a sus necesidades.

En general las fuentes de información de cualquier ámbito científico, se modifican a un ritmo vertiginoso: cambian sus versiones con frecuencia, incorporan novedades, surgen nuevos productos más específicos, desaparecen o modifican sus direcciones de acceso... por lo que resulta extremadamente difícil para cualquier investigador estar al tanto de todo ello y sobre todo conocer nuevos productos que pueden resultarle de especial interés en sus estudios.

La mayoría de las universidades españolas cuentan con buenos servicios bibliotecarios $\mathrm{y} / \mathrm{o}$ centros de documentación que tienen precisamente entre sus objetivos facilitar a los investigadores esta tarea; así se encargan de estudiar y seleccionar las fuentes, de contactar con productores y/o proveedores y de realizar los trámites administrativos necesarios para su consulta; al mismo tiempo suelen contar con profesionales especializados que están al tanto de los cambios y las novedades que se producen y conocen los nuevos productos y servicios ofrecidos por empresas e instituciones.

Todo ello hace patente en la actualidad la necesidad de estrechar la colaboración entre investigadores y bibliotecarios que permita a aquellos acceder a las fuentes más adecuadas en cada momento a sus proyectos, conocer las últimas actualizaciones y modificaciones 
$\mathrm{y}$, en definitiva, acercarse desde un campo específico de estudio a las nuevas posibilidades que el mundo de la documentación va proporcionando.

\section{BIBLIOGRAFÍA}

Aliaga Abad, F. y Suárez Rodríguez J. (1995). Las redes de ordenadores: nuevas herramientas para la investigación educativa. Relieve 1 (2). Recuperado el 7 de mayo de $2003 \quad$ de http://www.uv.es/RELIEVE/v1/RELIEVEv $\underline{\ln 2 . h t m}$.

Carrizo Sainero, G. (2000). Las fuentes de información. En G. Carrizo Sainero (ed.), Manual de fuentes de información (pp. 2355). Zaragoza: Confederación Española de Gremios y Asociaciones de Libreros.

Bradley, P. (2004). Internet tools for the advanced searcher. Recuperado el 31/03/04 de http://www.philb.com/adint.htm.

Fuentes i Pujol, M. .E. (1992). Documentación científica e información: metodología del trabajo intelectual y cientifico. Barcelona: PPU.
Herring, J. E. (2004). The Internet and information skills: a guide for teachers and school librarians. London: Facet Publishing.

Martínez López, F. J., Luna Huertas, P., Fernández Carrión, R. y Salmerón Silvera, J. L. (2001). Internet para investigadores: hacia la e-ciencia. Huelva: Universidad de Huelva.

Meira Cartea, P. (1997). Proyecto docente. Manuscrito no publicado, Santiago de Compostela.

Müller, J. F. (2003). A Librarian's guide to internet: searching and evaluating information. Oxford: Chandos.

Puig, J. (1987). Información pedagógica. En A. Sanvisens (ed.), Introducción a la pedagogía (pp. 425-444). Barcelona: Barcanova.

Sandín Esteban, M. P. (2001). Proyecto docente. Manuscrito no publicado, Universitat de Barcelona.

UNE 50-113-91/2: Documentación e información, vocabulario. Parte 2, Documentos tradicionales (1991). Recuperado de Suscrinorma.

\section{$\underline{\text { ABOUT THE AUTHORS / SOBRE LOS AUTORES }}$}

Alfonso Lorenzo Rodríguez (alfonso.lorenzo@altia.es). Doctor europeo en Ciencias Físicas (Universidade de Santiago, 1999).Colaborador del grupo de investigación Stellae de la Universidade de Santiago de Compostela. ALTIA Consultores S.L., c/Monte dos Postes 47, 15703 Santiago de Compostela.

Ana Belén Martínez Piñeiro (amp@uvigo.es). Bibliotecaria referencista de la Biblioteca Universitaria de Vigo; especializada en recursos de información y formación de usuarios. Universidade de Vigo, Biblioteca Central, Campus Lagoas-Marcosende, 36200 Vigo.

Esther Martínez Piñeiro (mpineiro@usc.es). Profesora titular del Departamento de Métodos de Investigación e Diagnóstico en Educación de la Universidade de Santiago. Responsable de la materia de Métodos de investigación socioeducativa de la titulación de Educación Social y miembro del grupo de investigación Stellae de dicha universidad. Ámbitos de trabajo: metodología de evaluación de programas socioeducativos, métodos de investigación socioeducativa. Universidade de Santiago de Compostela, Facultade de Educación, Campus universitario sur, 15782 Santiago de Compostela. 


\section{ARTICLE RECORD / FICHA DEL ARTÍCULO}

\begin{tabular}{|c|c|}
\hline $\begin{array}{l}\text { Reference / } \\
\text { Referencia }\end{array}$ & $\begin{array}{l}\text { Lorenzo Rodríguez, Alfonso Martínez Piñeiro, Ana Belén y Martínez Piñeiro, Esther (2004). Fuen- } \\
\text { tes de información en investigación socioeducativa. Revista ELectrónica de Investigación y EValuación } \\
\text { Educativa, v. } 10 \text {, n. 2. http://www.uv.es/RELIEVE/v10n2/RELIEVEv10n2_6.htmConsultado en } \\
(\text { poner fecha). }\end{array}$ \\
\hline Title / Título & Fuentes de información en investigación socioeducativa.[Information sources on socio-educational research] \\
\hline $\begin{array}{l}\text { Authors / Au- } \\
\text { tores }\end{array}$ & Alfonso Lorenzo, Ana Belén Martínez Piñeiro, Esther Martínez Piñeiro \\
\hline $\begin{array}{l}\text { Review / } \\
\text { Revista }\end{array}$ & Revista ELectrónica de Investigación y EValuación Educativa (RELIEVE), v. 10, n. 2 \\
\hline ISSN & $1134-4032$ \\
\hline $\begin{array}{l}\text { Publication } \\
\text { date / } \\
\text { Fecha de pu- } \\
\text { blicación }\end{array}$ & $\begin{array}{l}2004 \text { (Reception Date: } 2004 \text { June 10; Approval Date: } 2004 \text { Dec 03; Publication Date: } 2004 \\
\text { Dec 21) }\end{array}$ \\
\hline $\begin{array}{l}\text { Abstract / } \\
\text { Resumen }\end{array}$ & $\begin{array}{l}\text { The objective of this work is to be a reference tool for social-educational researchers and staff: a } \\
\text { selection of sources of useful information classified in four categories: catalogues, databases, } \\
\text { searchers and webs on Internet, and tools for communication between professional colleagues. } \\
\text { The electronic addresses necessary for localizing them, are included.. } \\
\text { Este trabajo pretende ser una herramienta de consulta para investigadores y profesionales de la } \\
\text { investigación social: una selección de fuentes de información útiles clasificadas en cuatro grupos: } \\
\text { catálogos, bases de datos, buscadores y webs en internet, e instrumentos para la comunicación con } \\
\text { otros profesionales. Incluye las direcciones electrónicas necesarias para la localización de las } \\
\text { fuentes }\end{array}$ \\
\hline $\begin{array}{l}\text { Keywords } \\
\text { Descriptores }\end{array}$ & $\begin{array}{l}\text { Citizenship education; Educational research; Social science research; Information sources; Ref- } \\
\text { erence materials; Research tools; Internet; Databases } \\
\text { Educación social; Investigación educativa; Investigación en ciencias sociales; Fuentes de infor- } \\
\text { mación; Materiales de referencia; Herramientas para la investigación; Internet; Bases de datos }\end{array}$ \\
\hline $\begin{array}{l}\text { Institution / } \\
\text { Institución }\end{array}$ & ALTIA Consultores S.L; Universidade de Vigo; Universidade de Santiago de Compostela (Spain) \\
\hline $\begin{array}{l}\text { Publication } \\
\text { site / } \\
\text { Dirección }\end{array}$ & http://www.uv.es/RELIEVE \\
\hline $\begin{array}{l}\text { Language / } \\
\text { Idioma }\end{array}$ & Español (Title, abstract and keywords in english ) \\
\hline
\end{tabular}

\section{Revista ELectrónica de Investigación y $\mathbf{E V}$ aluación Educativa (RELIEVE)}

$$
\text { [ ISSN: 1134-4032 ] }
$$

(C) Copyright, RELIEVE. Reproduction and distribution of this articles it is authorized if the content is no modified and their origin is indicated (RELIEVE Journal, volume, number and electronic address of the document).

(C) Copyright, RELIEVE. Se autoriza la reproducción y distribución de este artículo siempre que no se modifique el contenido y se indique su origen (RELIEVE, volumen, número y dirección electrónica del documento). 